OPEN ACCESS

Edited by:

Francesco DiMeco,

Carlo Besta Neurological Institute

Foundation (IRCCS), Italy

Reviewed by:

Antonio Silvani,

Carlo Besta Neurological Institute

Foundation (IRCCS), Italy Jürgen Beck,

University Hospital Freiburg, Germany

*Correspondence:

Marco Skardelly

marco.skardelly@med.unituebingen.de

Specialty section:

This article was submitted to Neuro-Oncology and

Neurosurgical Oncology,

a section of the journal

Frontiers in Oncology

Received: 08 August 2021 Accepted: 26 January 2022 Published: 16 February 2022

Citation:

Behling F, Rang J, Dangel E, Noell S, Renovanz M, Mäurer I, Schittenhelm J,

Bender B, Paulsen F, Brendel B,

Martus P, Gempt J, Barz M, Meyer B,

Tatagiba $M$ and Skardelly M (2022)

Complete and Incomplete Resection

for Progressive Glioblastoma Prolongs

Post-Progression Survival.

Front. Oncol. 12:755430.

doi: 10.3389/fonc.2022.755430

\section{Complete and Incomplete Resection for Progressive Glioblastoma Prolongs Post-Progression Survival}

\author{
Felix Behling ${ }^{1,2}$, Julia Rang ${ }^{1}$, Elena Dangel ${ }^{1}$, Susan Noell ${ }^{1,2}$, Mirjam Renovanz ${ }^{1,2,3}$, \\ Irina Mäurer ${ }^{2,3}$, Jens Schittenhelm ${ }^{2,4}$, Benjamin Bender ${ }^{2,5}$, Frank Paulsen ${ }^{6}$, \\ Bettina Brendel ${ }^{7}$, Peter Martus ${ }^{7}$, Jens Gempt ${ }^{8}$, Melanie Barz ${ }^{8}$, Bernhard Meyer ${ }^{8}$, \\ Marcos Tatagiba ${ }^{1,2,9}$ and Marco Skardelly ${ }^{1,2^{*}}$
}

1 Department of Neurosurgery, University Hospital Tuebingen, Eberhard Karls University Tuebingen, Tuebingen, Germany, ${ }^{2}$ Center for Neuro-Oncology, Comprehensive Cancer Center Tuebingen Stuttgart, University Hospital Tuebingen, Eberhard Karls University of Tuebingen, Tuebingen, Germany, ${ }^{3}$ Department of Neurology and Interdisciplinary Neuro-Oncology, University Hospital Tuebingen, Eberhard Karls University of Tübingen, Tuebingen, Germany, ${ }^{4}$ Institute of Pathology and Neuropathology, Division of Neuropathology, University Hospital Tuebingen, Eberhard Karls University Tuebingen, Tübingen, Germany, ${ }^{5}$ Department of Neuroradiology, University Hospital Tuebingen, Eberhard Karls University Tuebingen, Tuebingen, Germany, ${ }^{6}$ University Department of Radiation Oncology, University Hospital Tuebingen, Eberhard Karls University of Tuebingen, Tuebingen, Germany, ${ }^{7}$ Institute of Clinical Epidemiology and Applied Biometry, University Hospital Tuebingen, Eberhard Karls University Tuebingen, Tuebingen, Germany, ${ }^{8}$ Department of Neurosurgery, School of Medicine, Klinikum rechts der Isar, Technische Universität Muenchen, Muenchen, Germany, ${ }^{9}$ German Cancer Consortium (DKTK), Deutsche Krebsforschungszentrum (DKFZ) Partner Site Tuebingen, Tuebingen, Germany

Objective: The role of resection in progressive glioblastoma (GBM) to prolong survival is still controversial. The aim of this study was to determine 1) the predictors of postprogression survival (PPS) in progressive GBM and 2) which subgroups of patients would benefit from recurrent resection.

Methods: We have conducted a retrospective bicentric cohort study on isocitrate dehydrogenase $(I D H)$ wild-type GBM treated in our hospitals between 2006 and 2015. Kaplan-Maier analyses and univariable and multivariable Cox regressions were performed to identify predictors and their influence on PPS.

Results: Of 589 patients with progressive IDH wild-type GBM, 355 patients were included in analyses. Median PPS of all patients was 9 months (95\% Cl 8.0-10.0), with complete resection 12 months (95\% Cl 9.7-14.3, $\mathrm{n}=81$ ), incomplete resection 11 months (95\% Cl 8.9-13.1, $\mathrm{n}=70$ ) and without resection 7 months (95\% Cl 06-08, $\mathrm{n}=204)$. Multivariable Cox regression demonstrated a benefit for PPS with complete (HR 0.67, $\mathrm{Cl}$ 0.49-0.90) and incomplete resection (HR 0.73, 95\% Cl 0.51-1.04) and confirmed methylation of the 06-methylguanine-DNA-methyltransferase (MGMT) gene promoter, lower age at diagnosis, absence of deep brain and multilocular localization, higher Karnofsky Performance Status (KPS) and recurrent therapies to be associated with longer PPS. In contrast, traditional eloquence and duration of progression-free survival had no effect on PPS. Subgroup analyses showed that all subgroups of confirmed predictors benefited from resection, except for patients in poor condition with a KPS $<70$. 
Conclusions: Out data suggest a role for complete and incomplete recurrent resection in progressive GBM patients regardless of methylation of MGMT, age, or adjuvant therapy but not in patients with a poor clinical condition with a KPS $<70$.

Keywords: progressive glioblastoma, surgery, re-surgery, recurrent surgery, extent of resection, post progression survival, resectability

\section{INTRODUCTION}

Glioblastomas (GBM) are the most common and one of the most lethal malignant brain tumors with an incidence rate of 3-4 per 100,000 inhabitants (1). Despite multidisciplinary therapy with chemoradiotherapy, the prognosis remains poor with a median overall survival (OS) of about 16 months reported in recent studies (2-4).

At the time of tumor progression, patients undergo either local therapies such as recurrent surgery and radiotherapy and/ or systemic therapy depending on their age, symptom profile, general clinical condition and tumor localization. Resection is favored if the patient is in good clinical condition and the tumor is located in a well resectable area or if a significant progressive tumor mass is causing new neurological symptoms with impending escalation of intracranial pressure (5).

In GBM patients the resection of the primary tumor is associated with prolonged overall survival time (6) although the proposed resection thresholds range from maximum safe resection up to complete resection (7-11). In progressive GBM patients the role of recurrent resection is still controversial. While some studies did not demonstrate a benefit of recurrent resection at all $(12,13)$ others did (14-18). Age, Karnofsky Performance Status (KPS), tumor volume, extent of resection and eloquent tumor location were suggested as predictors of survival in GBM progression $(19,20)$. Several studies suggested complete tumor resection as an independent predictor of postprogression survival (PPS), while the role of incomplete resection is still controversial (15-18). Furthermore, currently a prospective trial is evaluating recurrent surgery in progressive glioblastoma (Schucht, clinicaltrials.gov). Nevertheless, only about 20-30 percent of patients with progressive GBM are considered for recurrent resection (21). It would be helpful in clinical routine to optimally select patients regarding probable benefit from recurrent surgery according to clinical factors.

However, as the published data remain contradictory and the resulting recommendations are still controversial, further research is warranted. Therefore, we conducted a retrospective bicentric cohort study with regard to the following topics: 1) Is there a role for resection in subgroups of progressive GBM patients; 2) do patients with progressive GBM benefit from incomplete tumor resection and 3) which subgroups of patients may benefit from resection in terms of age, KPS, $M G M T$ and other predictors of survival?

Abbreviations: GBM, Glioblastoma; IDH, Isocitrate dehydrogenase; KPS, Karnofsky performance status; MGMT, O6-methylguanine-DNAmethyltransferase; PFS, Progression free survival; PPS, Post-progression survival.

\section{METHODS}

\section{Study Design}

We conducted a retrospective bicentric cohort study on the importance of resection in patients with progressive isocitrate dehydrogenase $(I D H)$ wild-type GBM. Patients from two university hospitals (Universitaetsklinikum Tuebingen, BadenWuerttemberg, Germany and Klinikum rechts der Isar, Technische Universität Muenchen, Germany) were included. The main clinical endpoint was post-progression, as overall survival is biased by not including patients who died before first diagnosed progression, thus estimating too long an overall survival. Survival was evaluated by Kaplan-Meier analyses and univariable and multivariable Cox regressions.

\section{Study Population}

All patients (age $\geq 18$ years) with progressive $I D H$ wild-type glioblastoma who underwent surgery for the primary tumor between 2006 and 2015 in one of the participating centers were included in this study. The institutional ethics committees approved the study. We collected the following data from patients records for each patient: Age, gender, tumor site and tumor localization including eloquent brain regions, mutations of $I D H 1 / 2$ and methylation status of O6methylguanine-DNA-methyltransferase (MGMT), tumor infiltration of the ventricular wall/subependymal spread, postoperative extent of resection and adjuvant treatments (radiotherapy, chemotherapy, radiochemotherapy and best supportive care) after initial and recurrent surgery, postoperative neurological deficits, Karnofsky Performance Status, time of initial diagnosis, time of progression, last visit and death. Extent of resection was determined by a neuroradiologist and a neurosurgeon using magnetic resonance imaging within 72 hours after surgery; complete resection was defined as no residual contrast enhancement. Patients with missing data of important covariates were excluded.

\section{Ethical Approval}

All procedures were in accordance with the ethical standards of the institutional and/or national research committee and with the 1964 Helsinki declaration and its later amendments or comparable ethical standards. The present study was approved by the Ethics Committee of Tuebingen, Baden-Wuerttemberg, Germany, approval No. 115/2015BO2).

\section{Statistical Analyses}

We analyzed the clinical endpoints using PPS and OS, which were defined as the intervals between initial diagnosis or first tumor progression (PPS) and the patient's death (OS) or last 
clinical follow-up/control (censored). The patients were initially divided into three groups: A) patients with complete recurrent tumor resection; B) patients with incomplete recurrent tumor resection and $\mathrm{C}$ ) patients without recurrent tumor resection or biopsy. Group C was subdivided further (see below).

First univariable Cox regressions evaluated established prognostic covariates (age, MGMT status (methylated vs. unmethylated), first and recurrent therapies (radiochemotherapy vs radiotherapy vs chemotherapy), first extent of resection (complete vs. incomplete vs no resection/biopsy), KPS, progression free survival (PFS) and potentially prognostic covariates (tumor location, traditional eloquence (yes/no), subependymal spread (yes/no), duration of progression-freesurvival, use of steroids (yes/no)) in glioblastoma patients.

KPS was dichotomized by classification and regression tree (CART) analyses and adjusted to established threshold defined in the literature ( $\geq 70$ vs $<70$ ). A new covariate "resectability" as recently introduced by the authors (22) based on univariable Cox regressions of tumor location. All tumors were divided into good resectable and bad resectable according to their location by the authors. Glioblastomas seated in deep areas such as the diencephalon, thalamus, basal ganglia, brain stem as well as multicentric tumors were rated as bad resectable.

Univariable significant covariates were included in multivariable analyses with bidirectional elimination. Interactions between covariates were evaluated and significant interactions were addressed a) by establishing a composite score for extent of resection and resectability and b) by repeating multivariable analyses stratified to KPS between KPS and recurrent therapy modality. Following the introduction of the composite score (resection/resectability), group C was divided into group C1 including patients who had not been operated on with good resectable tumors and group C2) patients with bad resectable tumors (Figure 1). The results were expressed as hazard ratios (HR) with 95\% confidence intervals (CIs) and p-values. The goodness of fit of the model was determined by a Cox and Snell pseudo-R2 corrected for the number of covariates.

PPS and OS were analyzed by Kaplan Maier curves. Median survival times were shown in months and 95\% confidence intervals (CI). We performed subgroup analyses to compare PPS between

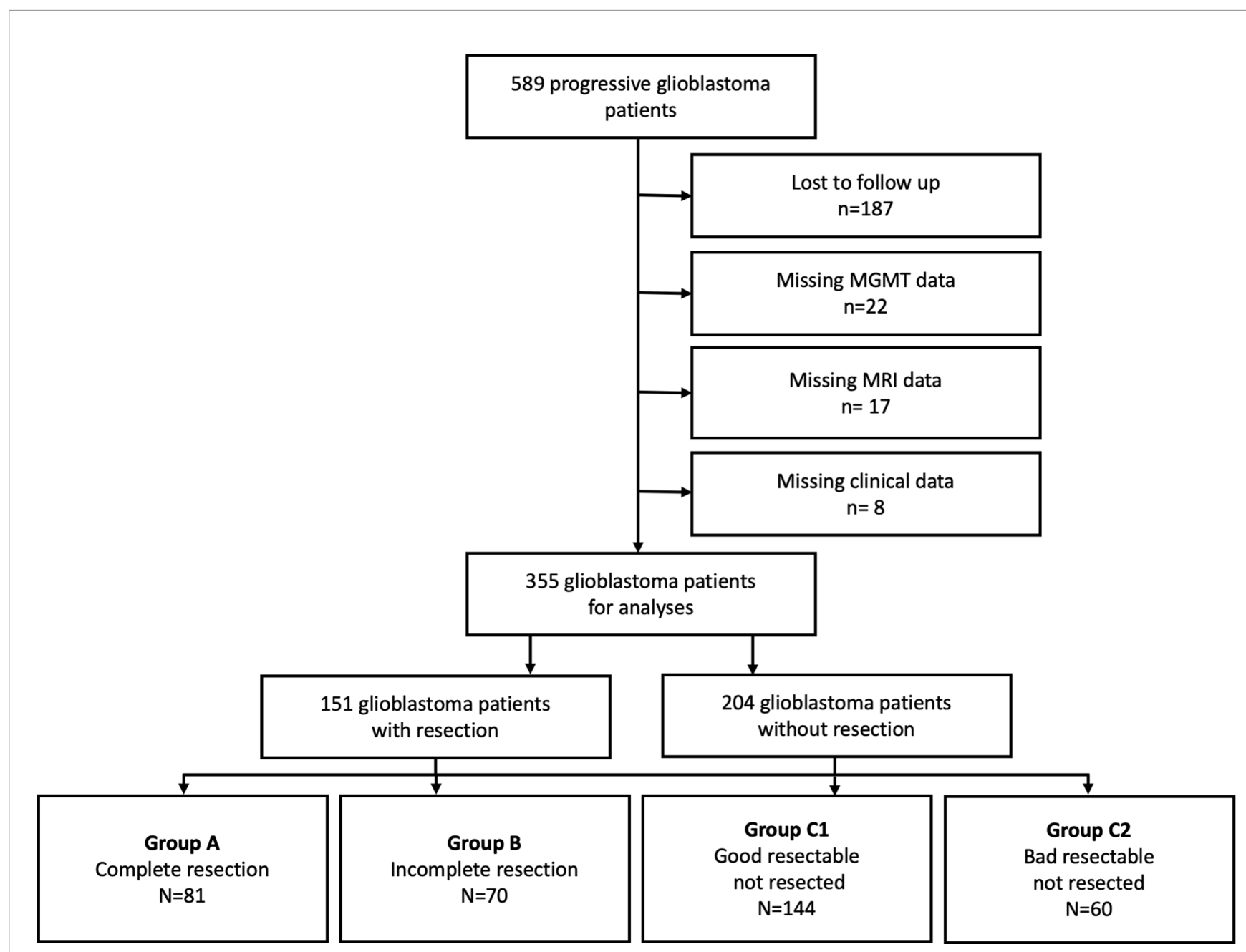

FIGURE 1 | Trial profile. Flow diagram of patients with progressive. IDH-Wildtype glioblastoma. 
patients with recurrent resection and without recurrent resection regarding methylation of the $M G M T$ promotor, age $(\leq 60,>60)$, KPS-Status ( $\leq 60,70-80,90-100)$, recurrent therapy (radiotherapy, chemotherapy, radiochemotherapy and no tumor specific therapy/ best supportive care) and progression free survival $(<3,3-6,6-12$, $>12$ ). These analyses were done after exclusion of patients with bad resectable (group C2) tumors to exclude the influence of tumor localization on Kaplan-Maier analyses (Figures 3A-D and Table 2).

The significance level was defined as $\alpha<0.05$ (two-sided), JMP $^{\circledR}$ (Cary, NC: SAS Institute Inc.; 1989) Statistical Discovery Software Version 14.2 and SPSS for Windows release 26 (Armonk, NY: IBM Corp 2019) was used for statistical analysis.

\section{RESULTS}

\section{Patients}

From 589 progressive $I D H$ wild-type GBM patients treated in our two centers from 2006 to 2015, we had complete data sets for multivariable analyses in 355 patients. Two hundred and thirty-four patients had to be excluded due to lost follow-up ( $n=187)$, missing MGMT promotor status $(\mathrm{n}=22)$ and missing magnetic resonance imaging data $(\mathrm{n}=17)$ or clinical data $(\mathrm{n}=8)$ (Figure 1). At the time of analysis $303(85 \%)$ patients had died and 52 (15\%) patients were either alive or no longer available for follow-up (censored data). The clinical data of the eligible patients are presented in Table $\mathbf{1}$ in relation to the 4 study groups A, B, C1 and C2. Eighty-one patients had a complete (group A) and 70 patients had an incomplete resection (group B). In group A, 54 patients (67\%) had stable KPS, 9 patients (11\%) had improved KPS, and 18 patients (22\%) had worsened KPS at discharge. In group B, 41 patients (59\%) had stable KPS, 9 patients (4\%) had improved KPS, and 18 patients (37\%) had worsened KPS at discharge. The median KPS change was 0 , and the 90/10 quantiles were 0 and $-20 \%$. The remaining 204 patients did not receive a resection. In 144 of these cases the lesion was assessed as good resectable (group C1) and in 60 cases as bad resectable (group C2). At disease progression, 204 patients received chemotherapy, 55 patients received radiotherapy, 28 patients received radio- and chemotherapy, and 68 patients received no therapy. After disease progression, most patients (103) received retreatment with temozolomide, either standard (5/28: 63 patients) or intensified protocol (7/7: 40 patients). Thirty-one patients received lomustine, 8 patients nimustine, and 14 patients other drugs or data not available in 47 patients.. Radiation therapy for progressive disease was highly variable and depended, among other factors, on the primary treatment, i.e., patients who had received first-line chemotherapy because of their age and methylation of MGMT received 34 Gy of radiotherapy in 10 fractions, and some received $60 \mathrm{~Gy}$ in 30 fractions. Other patients who had also received first-line radiotherapy received radiotherapy at a dose of $20 \mathrm{~Gy}$, for example, in $5 \times 4$ Gy or $4 \times 5$ Gy fractions as the disease progressed.

\section{Post Progression Survival, Overall Survival and Resectability}

Median PPS of all patients was 9 months (95\% CI 8.0-10). For patients who received a recurrent resection PPS was 11 months
(95\% CI 10-13, group A\&B)), with 12 months (95\% CI 9.7-14.3) for complete recurrent resection (group A) and 11 months (95\% CI 8.9-13.1) for incomplete recurrent resection (group B). Patients without a recurrent resection (group C) had a median PPS of 7 months (95\% CI 06-08) (Figure 2A). Sixty of 63 patients considered bad resectable according to the introduced resectability score were not operated on, 2 patients received an incomplete and 1 patient a complete resection. Median PPS was 8 months (95\% CI 6.9-9.1) in group C1 and 5 months (95\% CI 3.8-6.2) in group C2 (Figure 2B).

Subgroup analyses showed that patients benefited from resection independent of their methylation of MGMT promotor, age, duration of progression free survival and recurrence therapy

(Figures 3A, B, D and Table 2). In contrast, only patients with a KPS of $\geq 70$ benefited from re-resection. (Figure 3C and Table 2). In addition, patients older than 60 years who underwent recurrent resection showed a greater benefit in PPS than younger patients (median difference in PPS of 4 months vs 1 month) (Figure 3A and Table 2).

Median overall survival of all progressive patients was 17 months (95\% CI 15-18), for patients with complete recurrent resection (group A) 22 months (95\% CI 19-29), with incomplete recurrent resection (group B) 18 months (95\% CI 16-21) and for patients without recurrent resection for group C1 15 months (95\% CI 14-17) and group C2 13 months (95\% CI 11-16), respectively. Patients without progression within the observation period were not included in this study.

Some relevant clinical parameters were not well balanced between the study groups. Group C2 showed significant differences to the other groups regarding tumor localization, eloquence, subependymal spread, resectability, KPS due to the intrinsic concept of the variable "resectability". In addition, the age and frequency of initial and recurrent treatments were also not balanced between the groups, e.g. 32\% (group A), 19\% (group B), 47\% (group C1), and 22\% (group C2) of the patients were older than 65years (Table 1).

\section{Factors Associated With Benefit From Resection, Univariable Analysis}

Univariable Cox regressions suggested following covariates as significant predictors of PPS: recurrent resection (HR 0.61, 95\% CI 0.48-0.76, $\mathrm{p}<0.0001$ ); a) complete resection (risk ratio (HR) 0.55 (95\% CI 0.43-0.71, p<0.0001) and b) incomplete resection (HR 0.68, 95\% CI $0.51-0.91, \mathrm{p}<0.008)$ compared to no resection/biopsy; resectability (HR 0.53, 95\% CI 0.41-0.70, p<0.0001); methylated MGMT promotor (HR 0.67, 95\% CI 0.54-0.83, $\mathrm{p}=0.0002$ ); age at diagnosis (per decade decrease in HR 0.83 , 95\% CI 0.75-0.92, $\mathrm{p}=0.0002$; KPS $\geq 70$ (HR 0.42, 95\% CI 0.33-0.54, $\mathrm{p}<0.0001$ ), PFS (per month HR 0.97, 95\% CI 0.96-0.98, p<0.0001); absence of subependymal spread (HR 0.70, 95\% CI 0.57-0.87, p=0012); recurrent a) radiochemotherapy (HR 0.33 (95\% CI $0.36-0.77$, $\mathrm{p}<0.0001$ ), b) chemotherapy (HR 0.48 (95\% CI $0.37-0.62$, $\mathrm{p}<0.0001$ ) and c) radiotherapy (HR 0.73 (95\% CI 0.51-1.04, $\mathrm{p}=0.08$ ) compared to best supportive care in progressive disease. Resectability and extent of resection showed significant interaction $(\mathrm{p}=0.02)$ as well as KPS and recurrence therapy modality $(\mathrm{p}=0.003)$. 
TABLE 1 | Patients characteristics.

\begin{tabular}{|c|c|c|c|c|c|}
\hline & \multicolumn{5}{|c|}{ Resection/Resectability } \\
\hline Number of patients & $81(22 \%)$ & $70(18 \%)$ & $144(43 \%)$ & $60(17 \%)$ & $355(100 \%)$ \\
\hline female & 27 (33\%) & 24 (34\%) & $60(42 \%)$ & $20(33 \%)$ & $131(37 \%)$ \\
\hline male & $54(67 \%)$ & 46 (66\%) & $84(58 \%)$ & $40(67 \%)$ & $224(63 \%)$ \\
\hline \multicolumn{6}{|l|}{ Age } \\
\hline Quantile_75 & 69 & 63 & 64 & 69 & 75 \\
\hline Mean & 58 & 55 & 63 & 58 & 59 \\
\hline SD & 12 & 12 & 12 & 11 & 12 \\
\hline$\leq 50$ & $18(22 \%)$ & 25 (36\%) & $22(15 \%)$ & $11(18 \%)$ & $76(21 \%)$ \\
\hline$>50$ & $63(78 \%)$ & 45 (64\%) & $122(85 \%)$ & 49 (82\%) & 279 (79\%) \\
\hline$\leq 65$ & 55 (68\%) & 57 (81\%) & 77 (53\%) & 47 (78\%) & $236(66 \%)$ \\
\hline Biopsy & $2(2 \%)$ & $1(1 \%)$ & $14(10 \%)$ & $12(20 \%)$ & $29(8 \%)$ \\
\hline \multicolumn{6}{|l|}{ First therapy modality } \\
\hline Radiotherapy & $11(14 \%)$ & $8(11 \%)$ & $46(32 \%)$ & $11(18 \%)$ & $76(21 \%)$ \\
\hline Chemotherapy & 0 & $3(4 \%)$ & 19 (13\%) & 4 (7\%) & $26(7 \%)$ \\
\hline Radiochemotherapy & $61(75 \%)$ & 49 (70\%) & $62(43 \%)$ & $40(67 \%)$ & 212 (60\%) \\
\hline No therapy, study arm & $9(11 \%)$ & $10(14 \%)$ & $17(12 \%)$ & $5(8 \%)$ & 41 (12\%) \\
\hline \multicolumn{6}{|l|}{ MGMT } \\
\hline methylated & 25 (31\%) & 27 (39\%) & 59 (41\%) & $20(33 \%)$ & $131(37 \%)$ \\
\hline unmethylated & $56(69 \%)$ & $43(61 \%)$ & 85 (59\%) & $40(67 \%)$ & $224(63 \%)$ \\
\hline \multicolumn{6}{|l|}{ Side } \\
\hline right & $44(54 \%)$ & $36(51 \%)$ & $74(51 \%)$ & $31(52 \%)$ & $185(52 \%)$ \\
\hline left & 37 (46\%) & $34(49 \%)$ & 67 (47\%) & $22(37 \%)$ & $160(45 \%)$ \\
\hline bilateral & 0 & 0 & $3(2 \%)$ & 7 (12\%) & $10(3 \%)$ \\
\hline temporo-fronto-insular & $4(5 \%)$ & $5(7 \%)$ & $6(4 \%)$ & 0 & $15(4 \%)$ \\
\hline temporo-occipital & $3(4 \%)$ & $2(3 \%)$ & $6(4 \%)$ & 0 & $11(3 \%)$ \\
\hline occipital & $1(1 \%)$ & $3(4 \%)$ & $7(5 \%)$ & 0 & $11(3 \%)$ \\
\hline diencephalic & $1(1 \%)$ & 0 & 0 & $5(8 \%)$ & $6(1 \%)$ \\
\hline$>2$ lobes & $1(1 \%)$ & 0 & $11(7,5 \%)$ & 0 & $12(3 \%)$ \\
\hline multicentric & 0 & $2(3 \%)$ & $0 \%$ & 49 (82\%) & $51(15 \%)$ \\
\hline temporal & 27 (33\%) & $15(21 \%)$ & $39(27 \%)$ & 0 & $81(22 \%)$ \\
\hline insular & 0 & $1(1 \%)$ & $1(0,5 \%)$ & 0 & $2(1 \%)$ \\
\hline basal ganglia & 0 & 0 & 0 & $3(5 \%)$ & $3(1 \%)$ \\
\hline thalamic & 0 & 0 & 0 & $3(5 \%)$ & $3(1 \%)$ \\
\hline \multicolumn{6}{|l|}{ Tumor infiltrating ventricular wall } \\
\hline \multicolumn{6}{|l|}{ At progression } \\
\hline No & $50(63 \%)$ & 44 (63\%) & 84 (59\%) & 15 (25\%) & $193(55 \%)$ \\
\hline Yes & 31 (38\%) & 26 (37\%) & 59 (41\%) & 45 (75\%) & $160(45 \%)$ \\
\hline \multicolumn{6}{|l|}{ Resectability at progression } \\
\hline Bad & $1(1 \%)$ & $2(3 \%)$ & 0 & $60(100 \%)$ & $63(18 \%)$ \\
\hline Good & 80 (99\%) & $68(97 \%)$ & $144(100 \%)$ & 0 & $290(82 \%)$ \\
\hline \multicolumn{6}{|l|}{ Time to first progression } \\
\hline Median in months & 8 & 6 & 6 & 6 & 7 \\
\hline $95 \% \mathrm{Cl}$ in months & 6-9 & 4-8 & 4-8 & 6-7 & 6-7 \\
\hline$\leq$ 3months & $14(17 \%)$ & $24(34 \%)$ & $28(20 \%)$ & $12(20 \%)$ & $78(23 \%)$ \\
\hline$>$ 3months $-\leq 6$ months & $21(26 \%)$ & 13 (19\%) & $49(34 \%)$ & $12(20 \%)$ & $95(25 \%)$ \\
\hline
\end{tabular}


TABLE 1 | Continued

\begin{tabular}{|c|c|c|c|c|c|}
\hline & \multicolumn{5}{|c|}{ Resection/Resectability } \\
\hline$>$ 6months $-\leq 12$ mnths & $25(31 \%)$ & $18(26 \%)$ & $48(33 \%)$ & $24(40 \%)$ & $115(32 \%)$ \\
\hline \multicolumn{6}{|l|}{ Eloquence at progression } \\
\hline not eloquent & $42(53 \%)$ & $27(39 \%)$ & $82(57 \%)$ & $17(28 \%)$ & $168(47 \%)$ \\
\hline central (motoric/sensoric) & $15(18 \%)$ & $11(15 \%)$ & $22(15 \%)$ & $6(10 \%)$ & $54(15 \%)$ \\
\hline Inferior parietal lobule & $4(5 \%)$ & 0 & $6(4 \%)$ & $1(2 \%)$ & $11(3 \%)$ \\
\hline primary visual cortex & $5(6 \%)$ & $9(13 \%)$ & $4(3 \%)$ & $1(2 \%)$ & $19(5 \%)$ \\
\hline Deep brain & 0 & $2(3 \%)$ & $5(3 \%)$ & $31(51 \%)$ & $38(11 \%)$ \\
\hline \multicolumn{6}{|c|}{ Karnofsky performance status at progression } \\
\hline Median & 90 & 80 & 80 & 70 & 80 \\
\hline Quantile_25 & 80 & 70 & 62,5 & 50 & 70 \\
\hline Radiotherapy & $8(10 \%)$ & $11(16 \%)$ & 27 (19\%) & $9(15 \%)$ & $55(15,5 \%)$ \\
\hline Chemotherapy & 52 (64\%) & $26(37 \%)$ & 85 (59\%) & $41(68 \%)$ & $204(57,5 \%)$ \\
\hline Radiochemotherapy & 7 (9\%) & $14(20 \%)$ & $6(4 \%)$ & $1(2 \%)$ & $28(8 \%)$ \\
\hline No therapy, best supportive care & $14(17 \%)$ & $19(27 \%)$ & $26(18 \%)$ & $9(15 \%)$ & $68(19 \%)$ \\
\hline
\end{tabular}

A

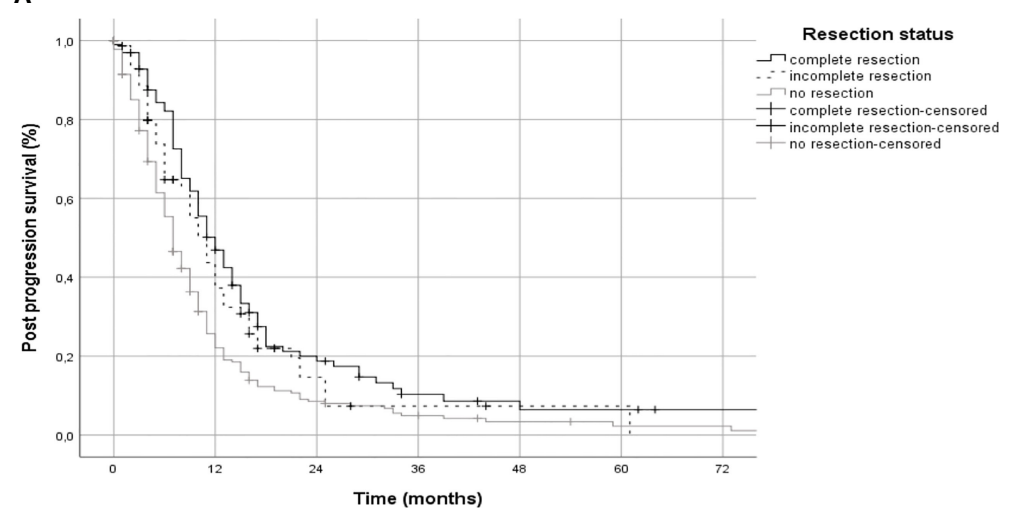

B

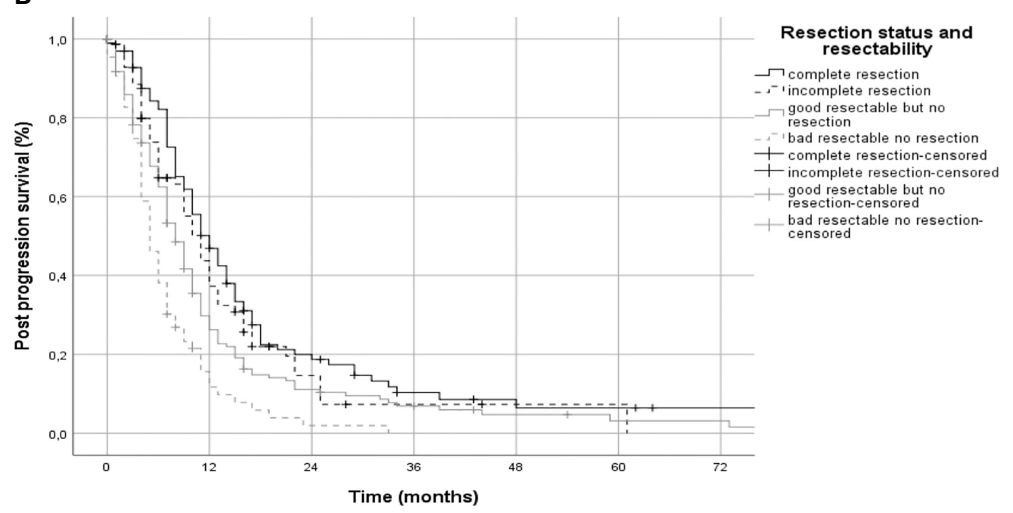

FIGURE 2 | Post-progression survival by extent of resection. Panel (A) shows post-progression survival in Kaplan-Meier estimates for patients stratified by complete, incomplete, and no resection. In panel (B) the group of patients who had no resection was divided into those with either good resectable or bad resectable tumors. 

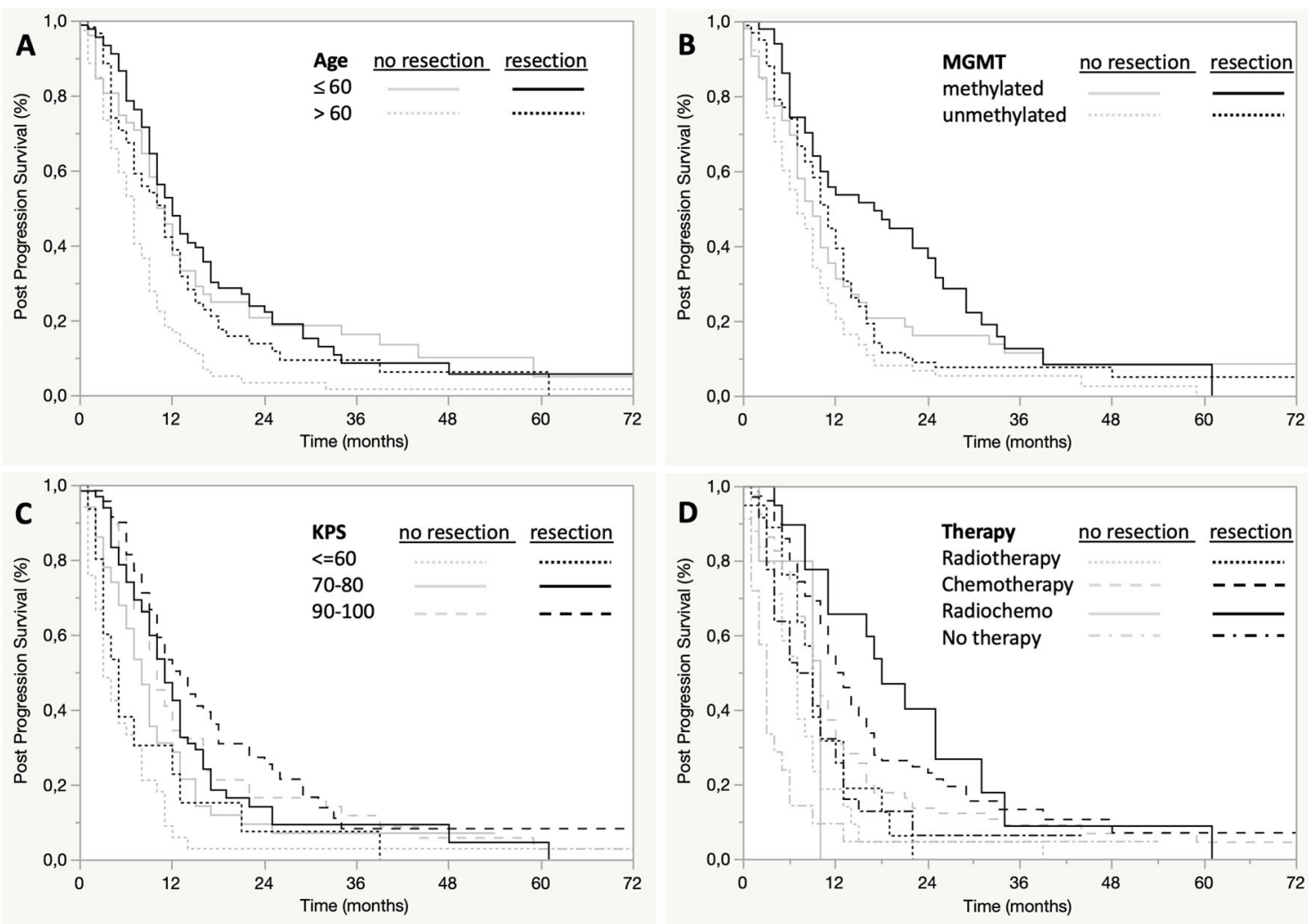

FIGURE 3 | Subgroup analysis of post-progression survival by age, MGMT, KPS, and adjuvant therapy. Post-progression survival is presented in Kaplan-Meier estimates, each bivariately stratified by extent of resection and age (A), MGMT methylation (B), KPS (C), or adjuvant therapy (D).

TABLE 2 | Post-progression survival as a function of recurrent resection performed and covariates.

\begin{tabular}{lcc}
\hline Resection & No & Yes \\
& & \\
\hline MGMT & & \\
$\quad$ methylated & & $17(9-25)$ \\
$\quad$ unmethylated & $9(7-11)$ & $11(9-12)$ \\
Age & $7(5-9)$ & $12(10-15)$ \\
$\leq 60$ & $11(8-13)$ & $11-(7-13)$ \\
$>60$ & $7(5-8)$ & \\
KPS & & \\
$\leq 60$ & $4(2-12)$ \\
$70-80$ & $8(6-9)$ & $11(9-13)$ \\
$90-100$ & $10(9-12)$ & $13(10-17)$ \\
Therapy & $7(4-9)$ & $9(5-13)$ \\
$\quad$ Radiotherapy & $10(8-11)$ & $13(11-15)$ \\
Chemotherapy & $10(2-10)$ & $18(11-25)$ \\
Radiochemotherapy & $3(1-4)$ & $8(4-10)$ \\
No therapy, best supportive care &
\end{tabular}

MGMT, O6-methylguanine-DNA-methyltransferase; KPS, Karnofsky Performance Status.

\section{Multivariable Analysis Regarding the Prognostic Factors}

The final multivariable Cox regression model $(\mathrm{R} 2=0.28$, Chi2 $=$ $117.696, \mathrm{n}=355$ ) showed a clear risk reduction for PPS after both complete resection (group A, HR 0.67, 95\% CI 0.49-0.90, $\mathrm{p}=0.009$ ) and incomplete resection (group B; HR 0.73, 95\% CI 0.51-1.04, $\mathrm{p}=0.08$ ) compared to no resection of good resectable tumors (group C1). Patients with bad resectable tumors showed 
an increased risk for death (group C2, HR 1.84, 95\% CI 1.31-2.17, $\mathrm{p}<0.0001)$ compared to patients with good resectable tumors who had no recurrent surgery (Table 3 ).

Furthermore, multivariable Cox regression confirmed the prognostic role of methylation of the MGMT gene promoter (HR $0.59, \mathrm{p}>0.0001$ ), age at diagnosis (per decade decrease, decrease in HR $0.85, \mathrm{p}=0.003$ ), KPS $\geq 70$ at progression (HR $0.23, \mathrm{p}<0.0001$ ) and recurrent a) radiochemotherapy (HR $0.11, \mathrm{p}=0.03), \mathrm{b})$ chemotherapy (HR 0.19, p<0.0001) and c) radiotherapy (HR 0.26, $\mathrm{p}=0.001$ ) at progression were associated with PPS (Table 3). Progression-free survival, subependymal spread and the traditional eloquent regions had no independent effect on PPS.

Post-progression survival, analyzed using Kaplan-Meier curves separately for patients with a favorable or unfavorable constellation of covariates (age, therapy, KPS and MGMT), showed different effects for patients with incomplete resection (Figures 4A, B). Patients with a favorable prognosis showed almost the same PPS with incomplete resection (11 months, CI 95\% 8-14) as patients with non-resected good resectable tumors (10 months, CI 95\% 8-12, Figure 4A). In contrast, patients with a poor prognosis with incomplete resection (9 months, CI 95\% 414) showed better PPS than patients with non-resected good resectable tumors (6 months, CI 95\% 5-7, Figure 4B).

\section{DISCUSSION}

The role of resection in progressive GBM patients remains controversial. The question arises, which subgroups of patients might benefit from recurrent resection in terms of extent of resection, age, KPS, PFS, recurrent therapies and molecular markers. We performed Kaplan-Meier and Cox regression analyses on a retrospective bicentric cohort of 355 progressive $I D H$ wild-type GBM patients and observed that patients in a good clinical condition (KPS) may benefit from complete and incomplete recurrent resection.

\section{Patients Population}

The median time to progression in our cohort was 7.0 months, which was very similar to the original study by Stupp et al. of 6.9 months (23) and the 7.1 months observed in study of 516 GBM patients by Helseth et al. (24) Median PPS was 9 months in our study, which is also in line with the 8.5 months observed by Helseth et al. (24).

\section{Association of Recurrent Resection and PPS}

In our study, patients who received recurrent surgery after tumor progression had a survival benefit of 4 months compared to patients who received no further surgery, which was also observed by Wann et al (14). We observed clear differences in PPS times between the different patient groups (A-C2). Patients with complete and incomplete resection showed a significant survival advantage with a risk reduction of approximately $30 \%$ with hazard ratios of 0.67 and 0.73 , respectively, in multivariable Cox regression compared to good resectable patients who did not receive recurrent surgery. Although statistical analysis narrowly missed significance for incomplete resection (HR 0.73, 95\% CI 0.51-1.04, $\mathrm{p}=0.08$, n.s.), the comparable HR of complete resection

TABLE 3 | Cox regression model.

\begin{tabular}{|c|c|c|c|c|c|}
\hline & HR & \multicolumn{2}{|c|}{$95 \% \mathrm{Cl}$} & df & $\mathbf{p}$ \\
\hline Reference: no resection but good resectable & & & & 3 & $<0.001$ \\
\hline Incomplete resection & 0.731 & 0.513 & 1.041 & 1 & 0.082 \\
\hline No resection, bad resectable & 1.839 & 1.310 & 2.581 & 1 & $<0.001$ \\
\hline MGMT-unmethylated & & & Reference & & \\
\hline KPS at progression $<70$ & & & Reference & & \\
\hline KPS at progression $\geq 70$ & 0.234 & 0.135 & 0.404 & 1 & $<0.001$ \\
\hline Reference: no therapy & & & & 3 & $<0.001$ \\
\hline Radiotherapy & 0.264 & 0.121 & 0.575 & 1 & 0.001 \\
\hline Chemotherapy & 0.192 & 0.109 & 0.337 & 1 & $<0.001$ \\
\hline Radiochemotherapy & 0.109 & 0.014 & 0.832 & 1 & 0.03 \\
\hline Radiotherapy & & & & & \\
\hline $\begin{array}{l}\text { KPS70 at progression * } \\
\text { Chemotherapy }\end{array}$ & 3.035 & 1.577 & 5.841 & 1 & 0.001 \\
\hline $\begin{array}{l}\text { KPS70 at progression * } \\
\text { Radiochemotherapy }\end{array}$ & 5.500 & 0.671 & 45.056 & 1 & 0.112 \\
\hline
\end{tabular}

MGMT, O6-methylquanine-DNA-methyltransferase; KPS, Karnofsky Performance Status. KPS70 at progression * therapy arm show the interactions between the patient's condition and the therapies used. 
A

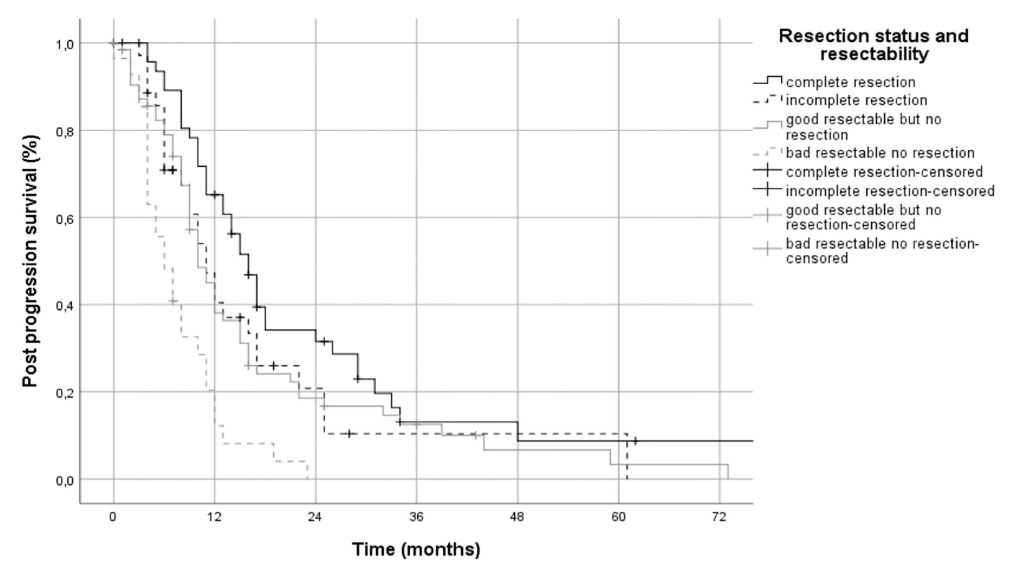

B

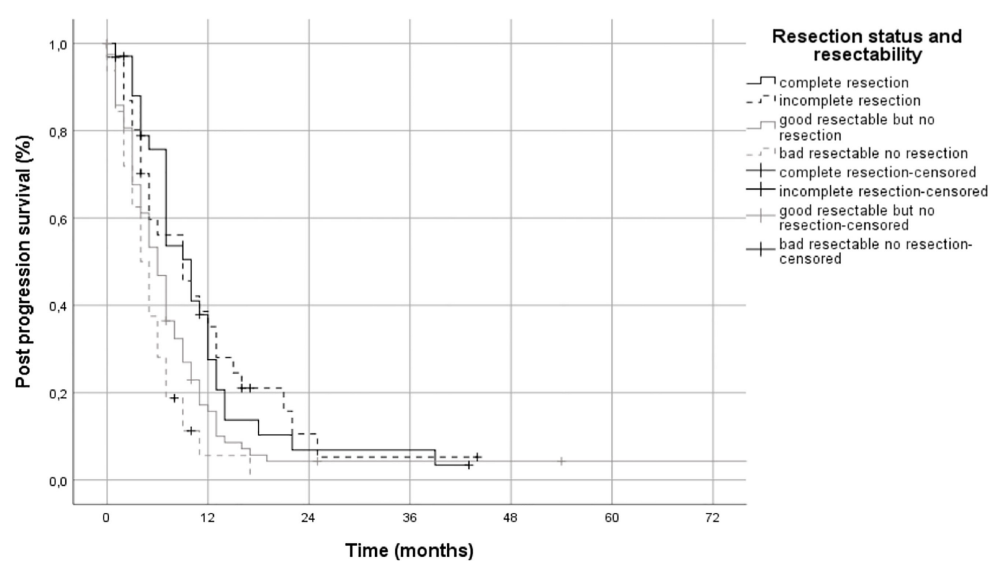

FIGURE 4 | Post-progression survival in patients with favorable and unfavorable constellation of covariates. Panel (A) shows post-progression survival in KaplanMeier estimates for patients with a favorable and panel (B) for an unfavorable constellation of the covariates age, MGMT, KPS, and adjuvant therapy stratified by extent of resection.

of 0.67 most likely suggests that it was missed due to an insufficient number of cases. This is in partial contrast to the retrospective observation of the prospective "DIRECTOR" study, which only showed a survival benefit for complete but not for incomplete tumor resection (15). Other studies also showed that not only complete tumor resection, but also a small residual tumor volume $\left(<3 \mathrm{~cm}^{3}\right)$ (16) or NTR (near total resection, i.e. only marginal enhancement of the resection cavity) (18) were associated with improved survival.

Based on our multivariable regression we investigated the effects of resection extent on PPS in patients with a favorable and a unfavorable covariate constellation (Figure 4). We found that patients with a favorable covariate constellation did not benefit from incomplete resection (group B, median PPS 11 months) compared to patients with good resectable tumors that were not resected (group C1, median PPS 10 months, Figure 4A), but patients with a unfavorable covariate constellation benefited from incomplete resection (group B, median PPS 9 months) compared to patients good resectable but not resected (group C1, median PPS 6 months, Figure 4B).

\section{Association of Progressive Tumor Location With PPS and KPS}

Traditional eloquent tumor localization has been proposed as an independent predictor of survival in progressive $\operatorname{GBM}(17,20)$. We could not confirm this observation when we applied electrophysiology and awake surgery in traditionally eloquent regions as we routinely do, but instead we identified a deep brain or multilocular location of the progressive tumor as an independent risk factor for shorter survival in our patient cohort, which we recently introduced as a new covariate that we called "resectability" (22). Despite routine electrophysiology and awake surgery to prevent new postoperative deficits, we observed new deficits at discharge in nearly $1 / 3(29 \%)$ of all patients, but in most cases they were mild (-10\% to $-20 \%)$. 
Nevertheless, the impact on quality of life from the risk of new deficits must also be considered when evaluating the chance of extending survival with recurrent resection.

\section{Association of Age, KPS, Recurrent Therapy and MGMT Methylation With Post-Progression Survival}

We confirmed age as a prognostic variable for PPS in patients with progressive GBM, but not as a predictive variable for benefit from recurrent surgery. With increasing age, PPS decreased, but also older patients showed a benefit of recurrent resection, as observed by Stark et al. (25). Furthermore, we observed that the median difference in PPS was even higher in re-resected older patients ( $>60$ years, 4 months) compared with patients who were not resected than in younger patients ( $<60$ years, 1 months) (Figure 3A and Table 2). This is probably due to a selection bias, since in progressive GBM $50 \%$ of younger patients ( $\leq 60$ years) were resected and only $35 \%$ of older patients ( $>60$ years). All subgroups of GBM patients stratified by MGMT, extent of first resection, therapies after progression and good KPS also benefited from recurrent resection with the exception of patients in poor clinical condition with a KPS $<70$. Therefore, only prognostic but not predictive roles for recurrent resection can be derived for these covariates.

\section{Impact of Progression Free Survival on Post-Progression Survival}

Dirks et al. reported 1993 that patients with GBM who had a recurrence later than 50 weeks after the first resection showed a significantly longer OS. PFS was therefore proposed as an independent variable for survival (26). We could not confirm duration of PFS as an independent predictor of PPS when the molecular markers $I D H$ and $M G M T$ were considered. Although GBM patients with PFS > 12 months also had longer PPS, this could be attributed to a more likely methylated MGMT promotor, e.g. the patient groups with $\leq 3$ months and $>12$ months PFS had a methylation rate of MGMT of $28 \%$ and $46 \%$. Since the established molecular biomarkers were not known by the time of the study by Dirks et al., it is more likely that the two groups stratified by PFS of 50 weeks had different rates of $I D H$-mutated and MGMT-methylated tumors.

\section{Limitations and Strengths of the Study}

The retrospective design is one of the main limitations of this study. Also, the determination of molecular markers such as mutation in the IDH gene or methylation of the MGMT promotor was not performed centrally according to a defined protocol, but locally in the two participating centers. Since tumor volumes were not determined by volumetry, but patients were stratified into groups with complete and incomplete resection or no resection at all, we had to limit the analyses of survival to semi-parametric methods and were not able to identify a possible threshold of residual tumor volume that would be associated with prolonged survival. Due to the retrospective design, the involved predictors of PPS are neither randomized nor stratified and are therefore not balanced in the investigated groups limiting the generalizability of the data. Nevertheless, our cohort of 355 patients with progressive $I D H$ wild-type GBM reflects the heterogeneity of the GBM patients in clinical routine and in our opinion the data represent this population in a realistic manner. Furthermore, the well-defined clinical and molecular data sets accounts for the uneven distribution of covariates to a large extent by performing multivariable regression including the covariates in addition to the univariable survival analyses.

\section{CONCLUSIONS}

1) Recurrent resection plays a distinct role in the therapy of selected progressive GBM patients

2) Our data suggest that both complete and incomplete resection may contribute to prolongation of PPS in selected progressive GBM patients.

3) Recurrent resection should be considered in patients with progressive GBM, regardless of age, methylation of MGMT or planned recurrent therapy presenting with KPS $\geq 70$ at the time of progression

4) In our patient cohort progression free survival and traditional eloquence were not associated with survival but tumors in a deep brain or multilocular location.

\section{DATA AVAILABILITY STATEMENT}

The raw data supporting the conclusions of this article will be made available by the authors, without undue reservation.

\section{ETHICS STATEMENT}

The studies involving human participants were reviewed and approved by Ethics Committee of Tuebingen, BadenWuerttemberg, Germany. Written informed consent for participation was not required for this study in accordance with the national legislation and the institutional requirements.

\section{AUTHOR CONTRIBUTIONS}

Conception and experimental design: FB and MS. Data acquisition: JR, ED, SN, IM, JS, BBe, FP, MR, JG, and MB. Analysis: MS, FB, BBr, and PM. Interpretation of the data: MS, FB, IM, JS, BBe, FP, BBr, PM, JR, MR, BM, and MT. Drafting: MS, FB, and PM. Revision: JR, ED, JS, IM, BBe, FP, MR, JG, MB, JS, BM, and MT. All authors contributed to the article and approved the submitted version.

\section{ACKNOWLEDGMENTS}

We acknowledge support by Open Access Publishing Fund of University of Tübingen. 


\section{REFERENCES}

1. Ostrom QT, Patil N, Cioffi G, Waite K, Kruchko C, Barnholtz-Sloan JS. CBTRUS Statistical Report: Primary Brain and Other Central Nervous System Tumors Diagnosed in the United States in 2013-2017. Neuro-Oncology (2020) 22:iv1-iv96. doi: 10.1093/neuonc/noaa200

2. Stupp R, Taillibert S, Kanner A, Read W, Steinberg DM, Lhermitte B, et al. Effect of Tumor-Treating Fields Plus Maintenance Temozolomide vs Maintenance Temozolomide Alone on Survival in Patients With Glioblastoma. JAMA (2017) 318:2306-11. doi: 10.1001/jama.2017.18718

3. Chinot OL, Wick W, Mason W, Henriksson R, Saran F, Nishikawa R, et al. Bevacizumab Plus Radiotherapy-Temozolomide for Newly Diagnosed Glioblastoma. N Engl J Med (2014) 370:709-22. doi: 10.1056/neimoa1308345

4. Gilbert MR, Dignam JJ, Armstrong TS, Wefel JS, Blumenthal DT, Vogelbaum MA, et al. A Randomized Trial of Bevacizumab for Newly Diagnosed Glioblastoma. N Engl J Med (2014) 370:699-708. doi: 10.1056/ nejmoa1308573

5. Wen PY, Weller M, Lee EQ, Alexander BM, Barnholtz-Sloan JS, Barthel FP, et al. Glioblastoma in Adults: A Society for Neuro-Oncology (SNO) and European Society of Neuro-Oncology (EANO) Consensus Review on Current Management and Future Directions. Neuro-Oncology (2020) 22:1073-113. doi: 10.1093/neuonc/noaa106

6. Brown TJ, Brennan MC, Li M, Church EW, Brandmeir NJ, Rakszawski KL, et al. Association of the Extent of Resection With Survival in Glioblastoma. JAMA Oncol (2016) 2:1460. doi: 10.1001/jamaoncol.2016.1373

7. Marko NF, Weil RJ, Schroeder JL, Lang FF, Suki D, Sawaya RE. Extent of Resection of Glioblastoma Revisited: Personalized Survival Modeling Facilitates More Accurate Survival Prediction and Supports a MaximumSafe-Resection Approach to Surgery. J Clin Oncol: Off J Am Soc Clin Oncol (2014) 32:774-82. doi: 10.1200/JCO.2013.51.8886

8. Chaichana KL, Jusue-Torres I, Navarro-Ramirez R, Raza SM, Pascual-Gallego M, Ibrahim A, et al. Establishing Percent Resection and Residual Volume Thresholds Affecting Survival and Recurrence for Patients With Newly Diagnosed Intracranial Glioblastoma. Neuro-oncology (2013) 16:113-22. doi: 10.1093/neuonc/not137

9. Kreth FW, Thon N, Simon M, Westphal M, Schackert G, Nikkhah G, et al. Gross Total But Not Incomplete Resection of Glioblastoma Prolongs Survival in the Era of Radiochemotherapy. Ann Oncol (2013) 24:3117-23. doi: 10.1093/annonc/mdt388

10. Sanai N, Polley M-Y, McDermott MW, Parsa AT, Berger MS. An Extent of Resection Threshold for Newly Diagnosed Glioblastomas. J Neurosurg (2011) 115:3-8. doi: 10.3171/2011.2.jns10998

11. Lacroix M, Abi-Said D, Fourney DR, Gokaslan ZL, Shi W, DeMonte F, et al. A Multivariate Analysis of 416 Patients With Glioblastoma Multiforme: Prognosis, Extent of Resection, and Survival. J Neurosurg (2001) 95:190-8. doi: 10.3171/jns.2001.95.2.0190

12. Nava F, Tramacere I, Fittipaldo A, Bruzzone MG, Dimeco F, Fariselli L, et al. Survival Effect of First- and Second-Line Treatments for Patients With Primary Glioblastoma: A Cohort Study From a Prospective Registry, 1997-2010. Neuro-Oncology (2014) 16:719-27. doi: 10.1093/ neuonc/not316

13. Clarke JL, Ennis MM, Yung WKA, Chang SM, Wen PY, Cloughesy TF, et al. Is Surgery at Progression a Prognostic Marker for Improved 6-Month Progression-Free Survival or Overall Survival for Patients With Recurrent Glioblastoma? Neuro-Oncology (2011) 13:1118-24. doi: 10.1093/neuonc/ nor110

14. Wann A, Tully PA, Barnes EH, Lwin Z, Jeffree R, Drummond KJ, et al. Outcomes After Second Surgery for Recurrent Glioblastoma: A Retrospective Case-Control Study. J Neuro-Oncol (2018) 137:409-15. doi: 10.1007/s11060017-2731-2
15. Suchorska B, Weller M, Tabatabai G, Senft C, Hau P, Sabel MC, et al. Complete Resection of Contrast-Enhancing Tumor Volume is Associated With Improved Survival in Recurrent Glioblastoma-Results From the DIRECTOR Trial. Neuro-Oncology (2015) 18(4):549-56. doi: 10.1093/ neuonc/nov326

16. Yong RL, Wu T, Mihatov N, Shen MJ, Brown MA, Zaghloul KA, et al. Residual Tumor Volume and Patient Survival Following Reoperation for Recurrent Glioblastoma. J Neurosurg (2014) 121:802-9. doi: 10.3171/ 2014.6.jns132038

17. Bloch O, Han SJ, Cha S, Sun MZ, Aghi MK, McDermott MW, et al. Impact of Extent of Resection for Recurrent Glioblastoma on Overall Survival. J Neurosurg (2012) 117:1032-8. doi: 10.3171/2012.9.jns12504

18. McGirt MJ, Chaichana KL, Gathinji M, Attenello FJ, Than K, Olivi A, et al. Independent Association of Extent of Resection With Survival in Patients With Malignant Brain Astrocytoma. J Neurosurg (2009) 110:156-62. doi: $10.3171 / 2008.4 .17536$

19. Hervey-Jumper SL, Berger MS. Reoperation for Recurrent High-Grade Glioma. Neurosurgery (2014) 75:491-9. doi: 10.1227/neu.0000000000000486

20. Park JK, Hodges T, Arko L, Shen M, Iacono DD, McNabb A, et al. Scale to Predict Survival After Surgery for Recurrent Glioblastoma Multiforme. J Clin Oncol (2010) 28:3838-43. doi: 10.1200/jco.2010.30.0582

21. Weller M, Cloughesy T, Perry JR, Wick W. Standards of Care for Treatment of Recurrent Glioblastoma-are We There Yet? Neuro-oncology (2013) 15:4-27. doi: 10.1093/neuonc/nos273

22. Skardelly M, Kaltenstadler M, Behling F, Mäurer I, Schittenhelm J, Bender B, et al. A Continuous Correlation Between Residual Tumor Volume and Survival Recommends Maximal Safe Resection in Glioblastoma Patients: A Nomogram for Clinical Decision Making and Reference for Non-Randomized Trials. Front Oncol (2021) 11:748691. doi: 10.3389/fonc.2021.748691

23. Stupp R, Mason WP, Bent MJvd, Weller M, Fisher B, Taphoorn MJB, et al. Radiotherapy Plus Concomitant and Adjuvant Temozolomide for Glioblastoma. N Engl J Med (2005) 352:987-96. doi: 10.1056/nejmoa043330

24. Helseth R, Helseth E, Johannesen TB, Langberg CW, Lote K, Rønning P, et al. Overall Survival, Prognostic Factors, and Repeated Surgery in a Consecutive Series of 516 Patients With Glioblastoma Multiforme. Acta Neurol Scand (2010) 122:159-67. doi: 10.1111/j.1600-0404.2010.01350.x

25. Stark AM, Hedderich J, Held-Feindt J, Mehdorn HM. Glioblastoma-the Consequences of Advanced Patient Age on Treatment and Survival. Neurosurg Rev (2006) 30:56-62. doi: 10.1007/s10143-006-0051-7

26. Dirks P, Bernstein M, Muller PJ, Tucker WS. The Value of Reoperation for Recurrent Glioblastoma. Can J Surg J Canadien Chir (1993) 36:271-5.

Conflict of Interest: The authors declare that the research was conducted in the absence of any commercial or financial relationships that could be construed as a potential conflict of interest.

Publisher's Note: All claims expressed in this article are solely those of the authors and do not necessarily represent those of their affiliated organizations, or those of the publisher, the editors and the reviewers. Any product that may be evaluated in this article, or claim that may be made by its manufacturer, is not guaranteed or endorsed by the publisher.

Copyright (๑) 2022 Behling, Rang, Dangel, Noell, Renovanz, Mäurer, Schittenhelm, Bender, Paulsen, Brendel, Martus, Gempt, Barz, Meyer, Tatagiba and Skardelly. This is an open-access article distributed under the terms of the Creative Commons Attribution License (CC BY). The use, distribution or reproduction in other forums is permitted, provided the original author(s) and the copyright owner(s) are credited and that the original publication in this journal is cited, in accordance with accepted academic practice. No use, distribution or reproduction is permitted which does not comply with these terms. 